\title{
Mammographie de dépistage: laissons les femmes choisir
}

Les recommandations du Swiss Medical Board concernant le dépistage par mammographie reposent sur une analyse médico-économique dont deux aspects sont discutables.

Thomas Perneger

Service d'épidémiologie clinique, Hôpitaux Universitaires de Genève Faculté de médecine, Université de Genève

Correspondance: Thomas Perneger, MD, PhD Service d'épidémiologie clinique,

Hôpitaux Universitaires de Genève

Rue Gabrielle-Perret-Gentil 4 CH-1211 Genève 4

thomas.perneger@hcuge.ch
Premièrement, cette analyse sous-évalue les bénéfices du dépistage. Selon le rapport, dans une cohorte de 10000 femmes dépistées pendant 13 ans, 16 évitent le décès et accumulent 80 années de vie ajustées

\section{Il est très simple de savoir ce qu'une femme en pense - il suffit de lui demander.}

pour la qualité. Mais à la fin de ce suivi, les femmes qui ont survécu grâce au dépistage ont encore une espérance de vie de quinze ou vingt ans. Les années de vie gagnées vont continuer à s'accumuler, alors que les coûts et les effets indésirables du dépistage auront cessé. tions supplémentaires, l'anxiété, ou l'inconfort. Leur importance peut être appréciée différemment d'une personne à l'autre. Prenons les résultats faussement positifs: environ une femme sur dix aura une mammographie faussement anormale une fois ou l'autre, et devra subir des examens complémentaires. Le SMB estime que sa qualité de vie diminue de $10 \%$ pendant 6 mois, ce qui équivaut à perdre 18 jours de vie en parfaite santé. Pour certaines femmes, cette appréciation peut être juste. Mais d'autres jugeront qu'un test anormal est un souci parmi d'autres, qui fait partie de la vie. Elles n'accepteraient pas de renoncer à 18 jours de leur existence pour éviter cet événement. Il est très simple de savoir ce qu'une femme en pense - il suffit de lui demander.

En résumé, le dépistage par mammographie peut faire gagner des années de vie, mais aussi causer des soucis réels. En vaut-il la peine? La réponse appar-

\section{Les femmes suisses ne pourront exprimer leurs préférences que si dépistage par mammographie demeure accessible.}

Deuxièmement, l'analyse des effets indésirables du dépistage ne tient pas compte des préférences individuelles. Ces effets indésirables sont des interven- tient à chaque femme concernée. Les femmes suisses ne pourront exprimer leurs préférences que si dépistage par mammographie demeure accessible. 